\title{
Scalable Synthesis of Crystalline One-Dimensional Carbon Nanothreads through Modest-Pressure Polymerization of Furan
}

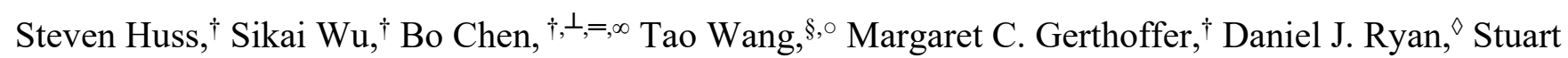

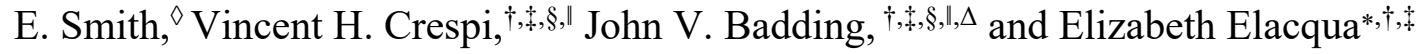

'Department of Chemistry, Pennsylvania State University, University Park, Pennsylvania 16802, United States

${ }^{*}$ Materials Research Institute, Pennsylvania State University, University Park, Pennsylvania 16802, United States

${ }^{\S}$ Department of Physics, Pennsylvania State University, University Park, Pennsylvania 16802, United States

'Department of Materials Science and Engineering, Pennsylvania State University, University Park, Pennsylvania 16802, United States

${ }^{\perp}$ Department of Chemistry and Chemical Biology, Cornell University, Baker Laboratory, Ithaca, New York 14853-1301, United States

${ }^{\bullet}$ ExxonMobil Research and Engineering Company, Annandale, New Jersey 08801, USA

= Donostia International Physics Center, Paseo Manuel de Lardizabal, 4, 20018 Donostia-San Sebastian, Spain

${ }^{\infty}$ IKERBASQUE, Basque Foundation for Science, Maria Diaz de Haro 3, 48013 Bilbao, Spain

${ }^{\circ}$ Department of Mechanical Engineering, The Pennsylvania State University, University Park, PA 16802, USA

${ }^{\Delta}$ Deceased October 26, 2019

\section{Supporting Information}

\section{Supporting Information (SI) Content:}

1. [4+2] Cycloaddition barrier calculations

2. Single-crystal X-ray diffraction of furan: solid-solid phase transition beginning

3. Computational methods: furan nanothreads cross-sections, packings, and X-ray diffraction

4. X-Ray photoelectron spectroscopy (XPS) on recovered solid from diamond anvil cell synthesis

5. IR Spectra simulations and comparison of peak positions with experiment

6. Raman spectrum of furan nanothreads produced in a PE Press

7. Reflective Infrared spectrum of furan nanothreads produced in a PE Press

8. 2-D diffraction from In-House XRD and WAXS of furan nanothreads produced in a PE Press

9. Infrared spectrum of recovered solid unexposed to air and exposed to air

10. Broadband FT-ICR MS of furan nanothreads produced from a PE Press 


\section{1. $[4+2]$ cycloaddition barrier calculations}

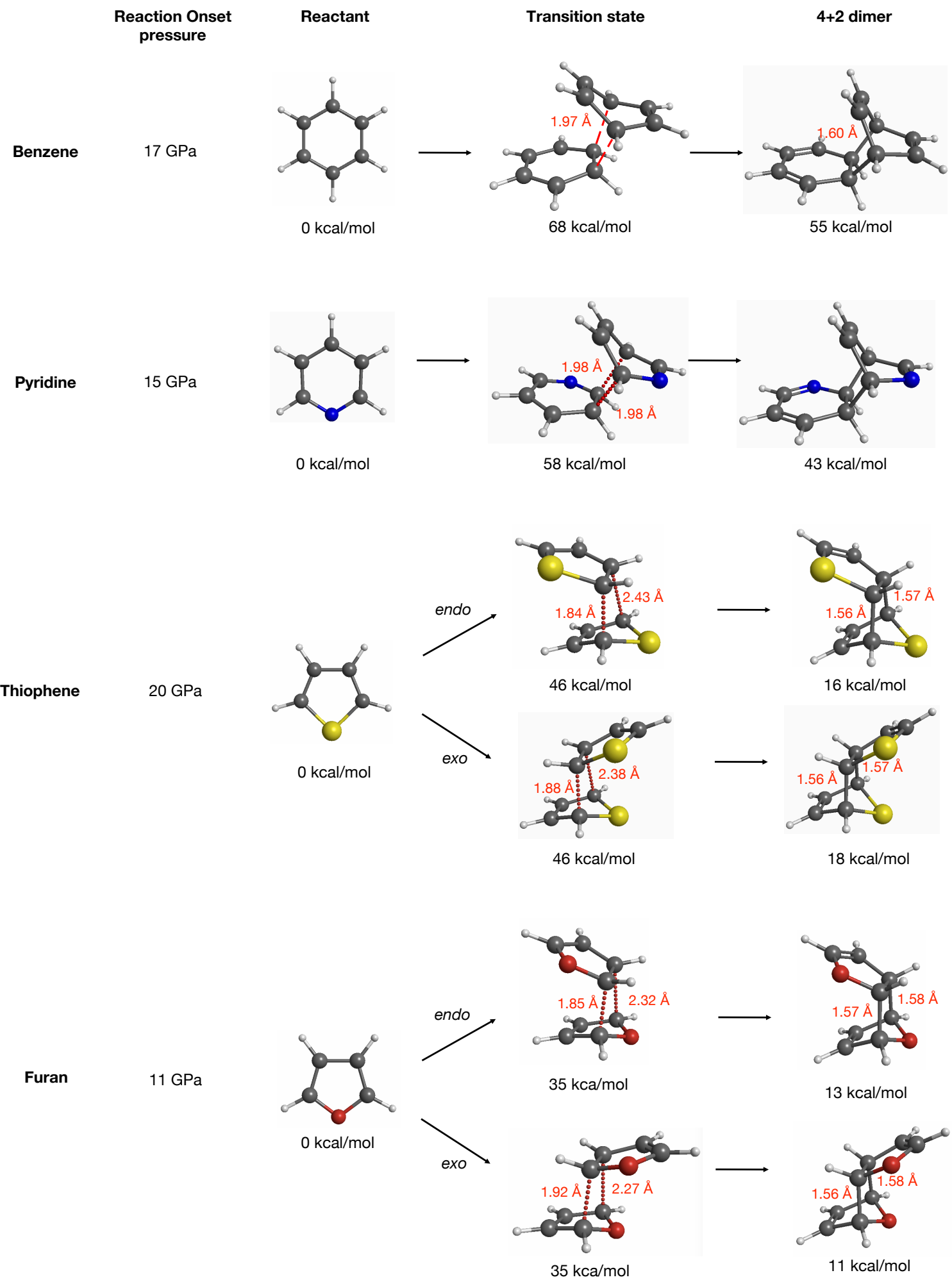

Figure S1. [4+2] Cycloaddition barriers and reaction energies for benzene, thiophene, and furan, computed at the B3LYP/6-31G(d) level of theory. The lowest energy transition state and dimer (out of 18 isomers ${ }^{1}$ for pyridine cycloaddition were shown. 


\section{Single-crystal X-ray diffraction of furan: Solid-solid phase transition beginning}
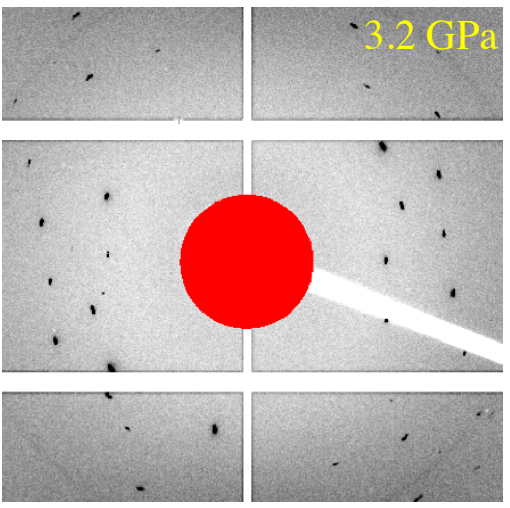
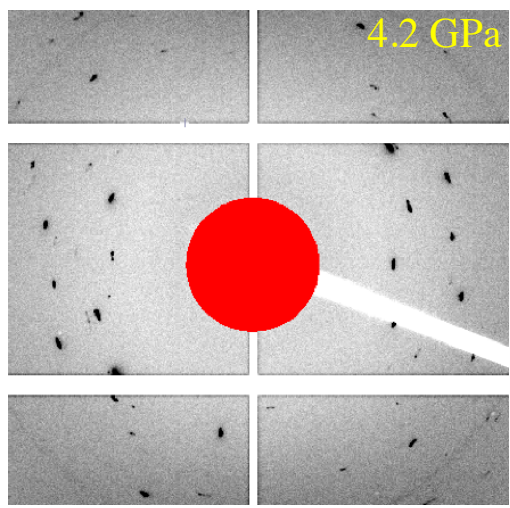
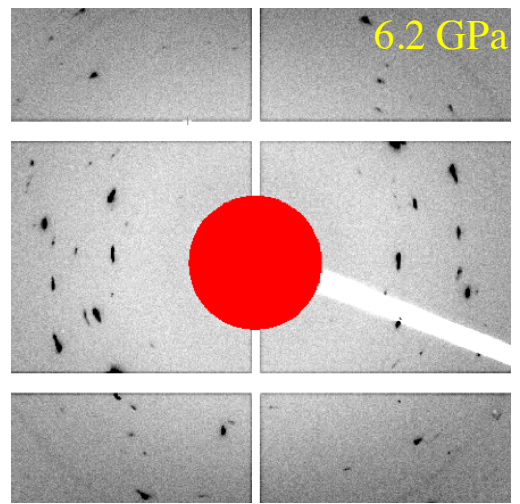

Figure S2. In situ synchrotron X-ray diffraction of furan single crystal reveals a phase transition beginning at ca. 4.2 GPa with the onset of new reflections. Red circle is a mask for the beam stop. 


\section{Computational methods: furan nanothreads cross-sections, packings, and X-ray diffraction}

Furan nanothreads do not have (quasi)circular cross sections as benzene-based nanothreads do. The cross-section shape of the syn thread has $\mathrm{C}_{2 \mathrm{v}}$ symmetry (Figure S3), while the cross-section shapes of the anti and syn-anti threads have $\mathrm{D}_{2 \mathrm{~h}}$ symmetry, the same symmetry as an ellipse. We treated the packing of furan threads in a solid as the $2 \mathrm{D}$ packing of an ellipse,${ }^{2}$ being fully aware that the ellipse approximation for the cross section may be less reasonable for the syn thread. There are only three dense packing patterns (each ellipse has 6 touching neighbors), categorized by the number of orientations of the ellipses. Type A has only one orientation (i.e., all ellipses have their long axes aligned in the same direction). Type B has two orientations, and the long axes of the ellipses with different orientations are not parallel, but at an angle. Type $\mathrm{C}$ has three orientations, with the long axes of the ellipses of different orientations being at 120-degree angles. Within each packing pattern, there are subtypes depending on where two neighboring ellipses touch (for type A and C), or the relative orientations of the ellipses (for type B).

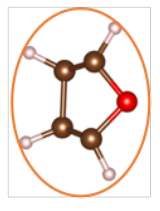

syn

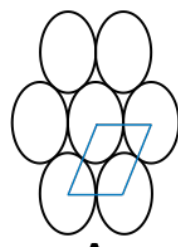

A

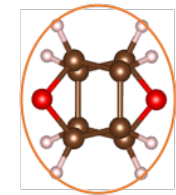

anti

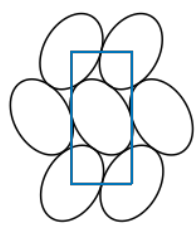

B

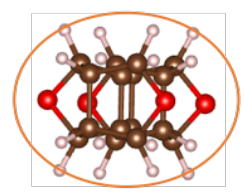

syn-anti

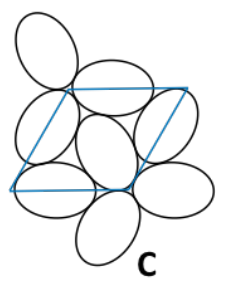

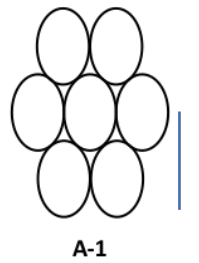

A-1

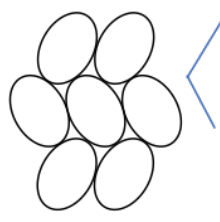

B-1

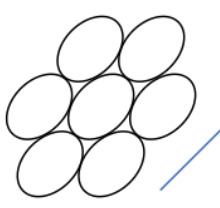

A-2

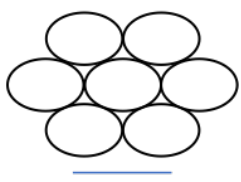

A-3

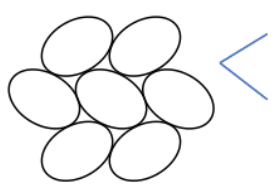

B-2

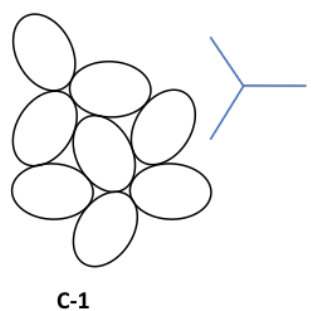

C-2

Figure S3. Left: Furan threads cross section shapes and three simple 2D dense packings of ellipse considered in this work. Unit cells for each packing are shown. Right: Subtypes of packing. The blue lines next to the patterns indicate the orientations of the long axes of the ellipses.

Note that there can be more complex patterns with large unit cell sizes. For example, a unit cell can have three ellipses, where two of the ellipses have the same orientation and the third ellipse has a different orientation. This packing belongs to type B because it has two orientations. We constructed the packings of furan nanothreads based on the seven subtypes in Figure S3. However, in the relaxed structures, the packing pattern can be quite different from the initial ones. Axial offsets between neighboring threads were also considered for some packing types. Axial offset or shift measures the distance (relative to the unit cell length) shifted by the second thread along the thread direction relative to the first thread. In this 
case of Figure S4 for the syn-anti thread, an axial shift of 0.5 (meaning shifted by a half unit cell) brings the oxygens on different threads closer to each other than in the packing without axial shift.

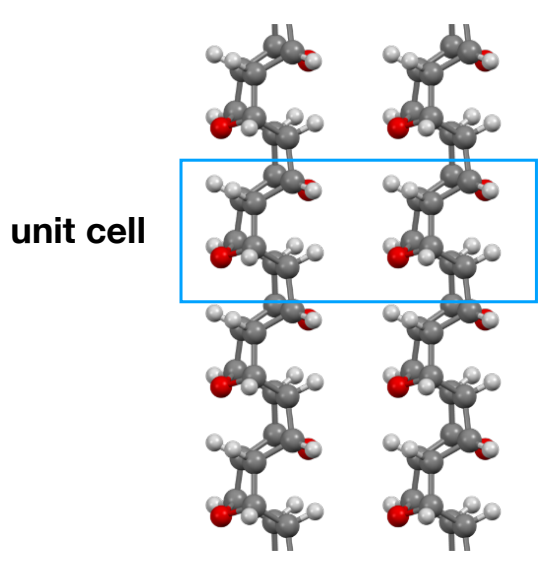

Axial shift $=0$

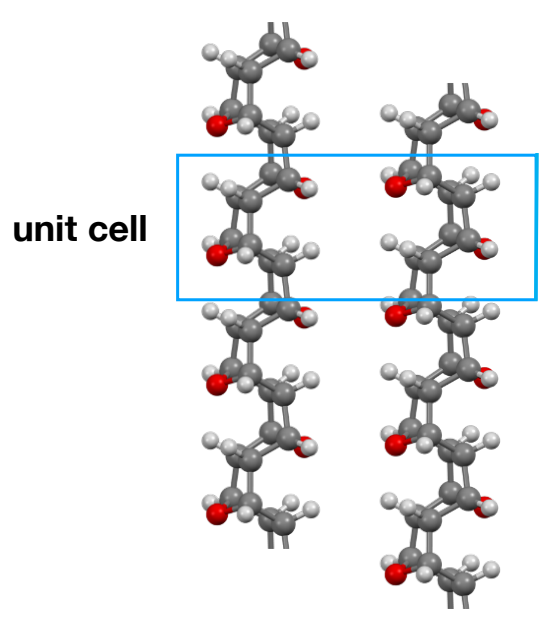

Axial shift $=0.5$

Figure S4. Axial shift.

Table S1 summarizes the relative energies, volumes and simulated d-spacings for furan nanothreads at $1.5 \mathrm{GPa}$. In the names (e.g., A-1-a), the first letter denotes the packing type, the second number denotes the subtype, and the third letter indicates different axial offsets. Figure S5 shows some of the simulated packings.

Table S1. Calculated relative enthalpies per furan unit (relative to B-3-b), volumes per furan unit, and dspacings of packed furan nanothreads of the syn, anti, and syn-anti configurations at $1.5 \mathrm{GPa}$. Calculations were done using the PBE functional, $600 \mathrm{eV}$ cutoff, and with the D3-BJ vdW correction. The lowest enthalpies, smallest volumes, and d-spacings closest to experimental values in each table are in red.

\begin{tabular}{|c|c|c|c|c|c|}
\hline syn & $\mathbf{H} / \mathbf{C}_{\mathbf{4}} \mathbf{H}_{\mathbf{4}} \mathbf{O}(\mathbf{e V})$ & $\mathbf{V} / \mathbf{C}_{\mathbf{4}} \mathbf{H}_{\mathbf{4}} \mathbf{O}\left(\mathbf{\AA}^{\mathbf{3}}\right)$ & \multicolumn{3}{|c|}{$d$-spacings $(\AA)$} \\
\hline $\mathbf{A - 1 - a}$ & 0.12 & 123 & 4.23 & 4.23 & 5.18 \\
\hline $\mathbf{A - 1 - b}$ & 0.18 & 126 & 4.20 & 4.30 & 5.33 \\
\hline $\mathbf{A - 2}-\mathbf{a}$ & 0.13 & 123 & 4.23 & 4.29 & 5.15 \\
\hline $\mathbf{A - 2 - b}$ & 0.23 & 130 & 4.38 & 4.42 & 5.21 \\
\hline $\mathbf{A - 3 - a}$ & 0.17 & 127 & 4.20 & 4.81 & 4.83 \\
\hline $\mathbf{A - 3 - b}$ & 0.14 & 125 & 4.36 & 4.62 & 4.83 \\
\hline
\end{tabular}




\begin{tabular}{|c|c|c|c|c|c|}
\hline B-1-a* & 0.10 & 123 & 4.20 & 4.69 & 5.10 \\
\hline B-1-b* & 0.13 & 124 & 4.26 & 4.29 & 5.17 \\
\hline B-2-a* & 0.28 & 137 & 4.48 & 4.91 & 5.02 \\
\hline B-2-b* & 0.09 & 125 & 2.17 & 2.17 & 4.11 \\
\hline B-3-a* & 0.03 & 123 & 2.27 & 4.85 & 4.49 \\
\hline B-3-b* & 0.00 & 122 & 2.20 & 2.21 & 4.35 \\
\hline B-4-a* & 0.09 & 125 & 2.17 & 2.17 & 4.13 \\
\hline B-4-b* & 0.08 & 124 & 2.16 & 4.19 & 4.52 \\
\hline C-1-a & 0.12 & 126 & 4.57 & 4.63 & 4.63 \\
\hline
\end{tabular}

* These packings show diffraction patterns that are far away from the observed quasi-hexagonal pattern. The d-spacings listed here are for the three pairs of spots with the largest intensities.

\begin{tabular}{|c|c|c|c|c|c|}
\hline anti & $\mathbf{H} / \mathbf{C}_{4} \mathbf{H}_{4} \mathbf{O}(\mathbf{e V})$ & $\mathbf{V} / \mathbf{C}_{4} \mathbf{H}_{4} \mathbf{O}\left(\AA^{3}\right)$ & \multicolumn{3}{|c|}{$d$-spacings $(\AA)$} \\
\hline A-1-a & 0.13 & 127 & 4.75 & 4.75 & 5.30 \\
\hline A-1-b & 0.14 & 127 & 4.72 & 4.75 & 5.28 \\
\hline A-2-a & -0.02 & 128 & 4.76 & 5.05 & 5.05 \\
\hline A-2-b & -0.06 & 128 & 4.74 & 5.04 & 5.06 \\
\hline B-1-a & -0.06 & 125 & 4.87 & 4.92 & 4.92 \\
\hline B-1-b & -0.07 & 125 & 4.79 & 4.88 & 5.02 \\
\hline B-2-a & -0.07 & 125 & 4.68 & 5.01 & 5.01 \\
\hline B-2-b & -0.15 & 122 & 4.79 & 4.85 & 4.89 \\
\hline C-1-a & -0.03 & 125 & 4.84 & 4.90 & 4.93 \\
\hline C-2-a & -0.07 & 123 & 4.77 & 4.88 & 4.91 \\
\hline C-3-a & 0.02 & 125 & 4.81 & 4.91 & 4.96 \\
\hline
\end{tabular}




\begin{tabular}{|c|c|c|c|c|c|}
\hline syn-anti & $\mathrm{H} / \mathrm{C}_{4} \mathrm{H}_{4} \mathbf{O}(\mathbf{e V})$ & $\mathrm{V} / \mathrm{C}_{4} \mathbf{H}_{4} \mathbf{O}\left(\AA^{\mathbf{3}}\right)$ & \multicolumn{3}{|c|}{$d$-spacings $(\AA)$} \\
\hline A-1-a & 0.16 & 130 & 4.77 & 4.78 & 5.45 \\
\hline A-2-a & -0.10 & 126 & 4.81 & 5.02 & 5.01 \\
\hline B-1-a & -0.11 & 125 & 4.91 & 4.93 & 4.94 \\
\hline B-2-a & -0.02 & 129 & 4.88 & 4.89 & 5.16 \\
\hline C-1-a & 0.12 & 136 & 4.96 & 5.19 & 5.23 \\
\hline C-2-a & 0.04 & 129 & 4.90 & 4.94 & 5.11 \\
\hline
\end{tabular}

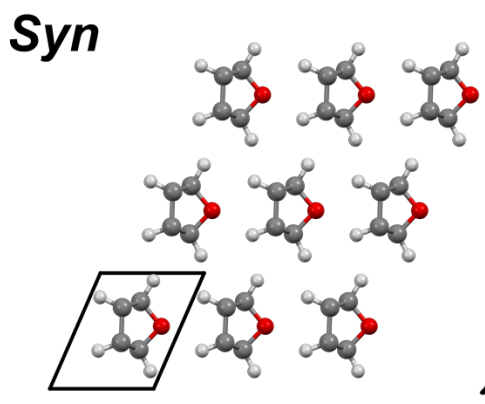

A-1

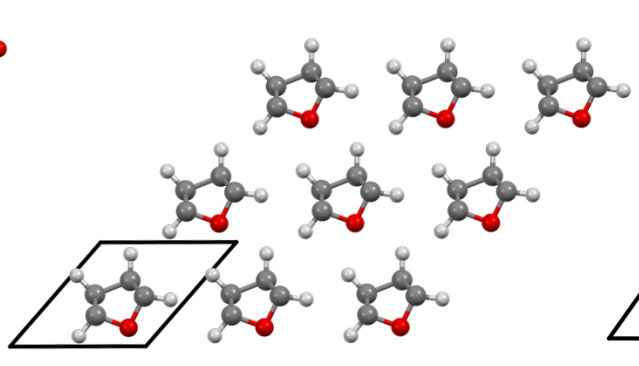

A-2

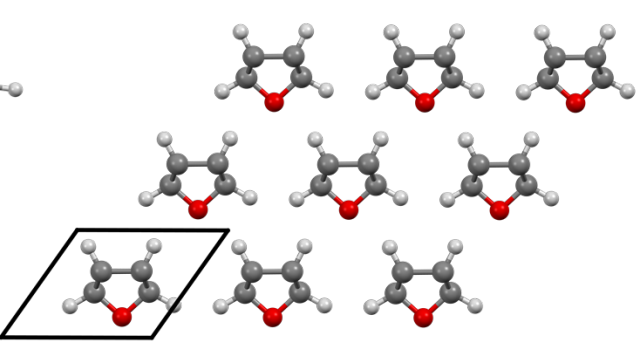

A-3

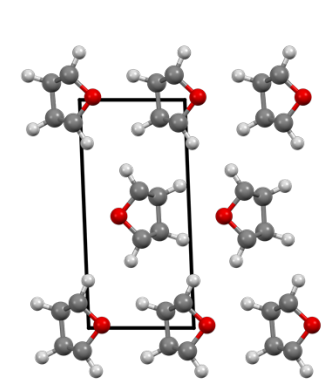

B-1

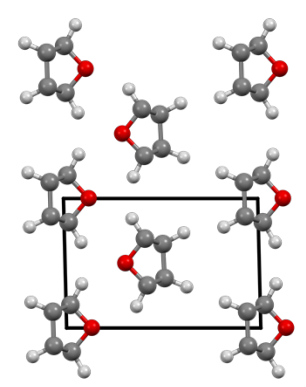

B-2

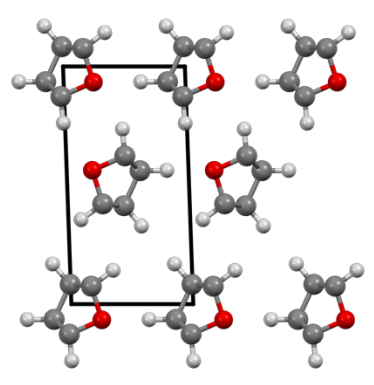

B-3

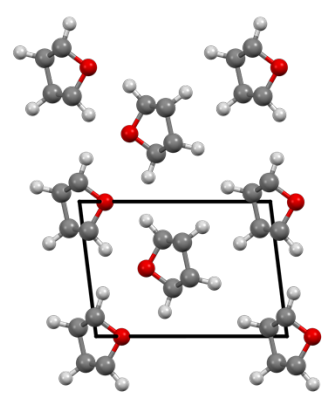

B-4

Figure S5. Simulated packings for syn furan threads. 


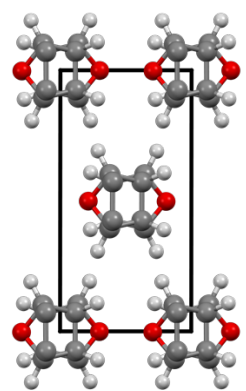

A-1

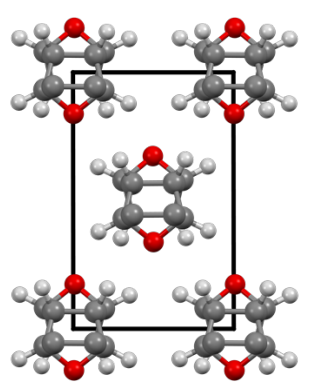

A-2

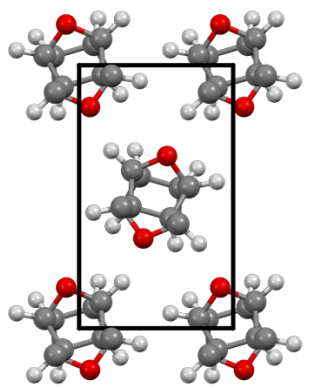

B-1

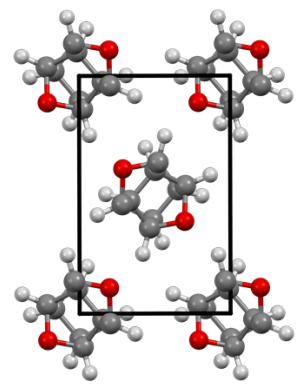

B-2

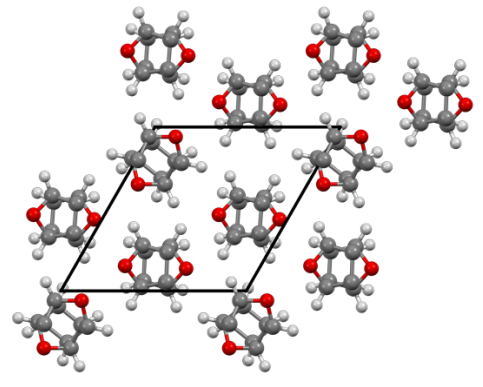

C-1

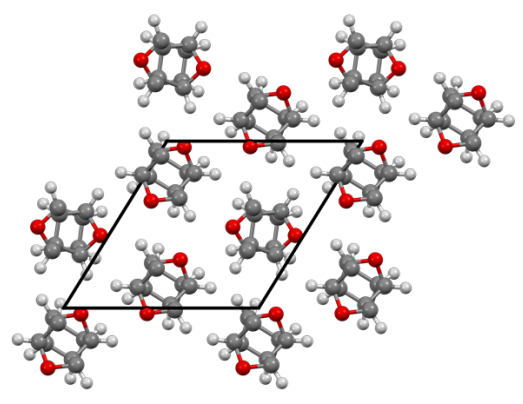

C-2

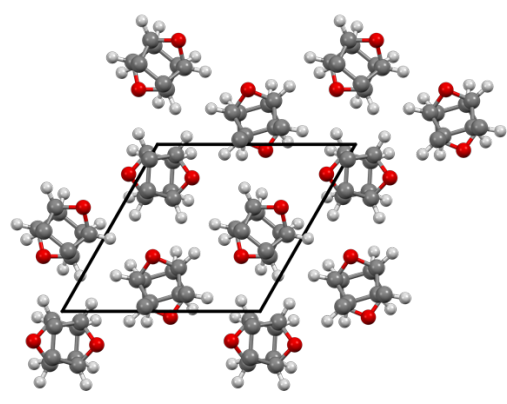

C-3

Figure S6. Simulated packings for anti furan threads.

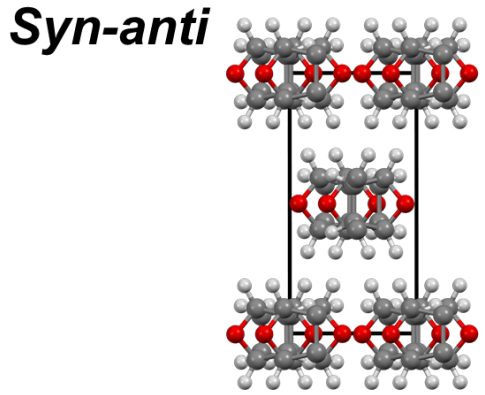

A-1

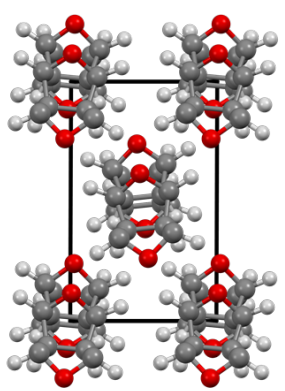

A-2

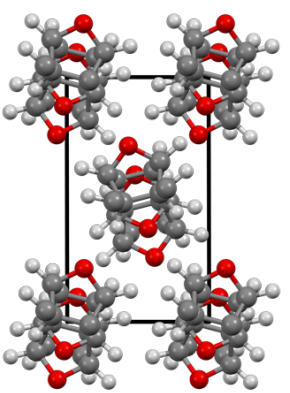

B-1

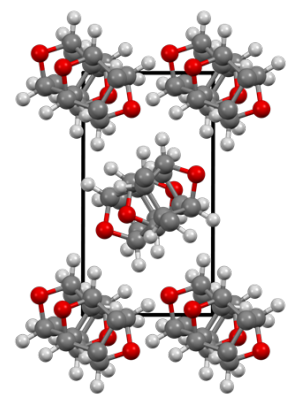

B-2

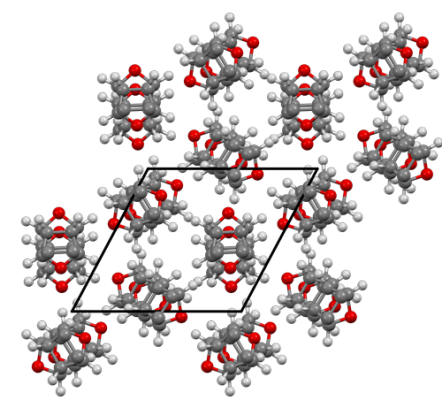

C-1

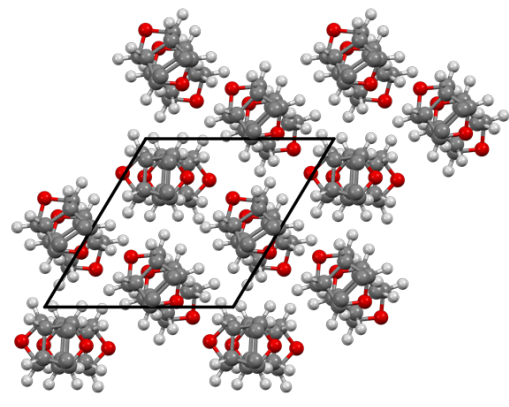

C-2

Figure S7. Simulated packings for syn/anti furan threads. 
Calculations show that the lowest enthalpy packing for the syn thread at $1.5 \mathrm{GPa}$ is B-3-b, for anti B2-b, and for syn-anti B-1-a. Among these three, B-2-b has the lowest enthalpy.

For the syn threads, since each individual thread (when straight) has a dipole moment in the transverse direction, the A type packings will result in a permanent dipole moment for the crystal because of the same orientation for all the threads. For the B and $\mathrm{C}$ type packings with multiple orientations, the dipole moments of individual threads can be aligned to achieve favorable dipole-dipole interaction, which might contribute to B-3-b being the lowest enthalpy packing. Of course, the small volume of B-3-b is another contributor.

The anti and syn-anti threads do not have dipole moments in the transverse direction. The calculated d-spacings of the C-2-a packing of the anti-thread has the best match to experimental values. The simulated diffraction pattern for this thread is shown in Figure S6 and compared with the experimental diffraction pattern. Not only the d-spacings but also the angles between the diffraction spots match well between simulation and experiment. Note that the calculations were done at $0 \mathrm{~K}$ temperature. If thermal expansion is considered, the calculated d-spacing is expected to expand by $1-3 \%$ at $300 \mathrm{~K}$ at which the experiment measurement was taken.

\section{experiment}

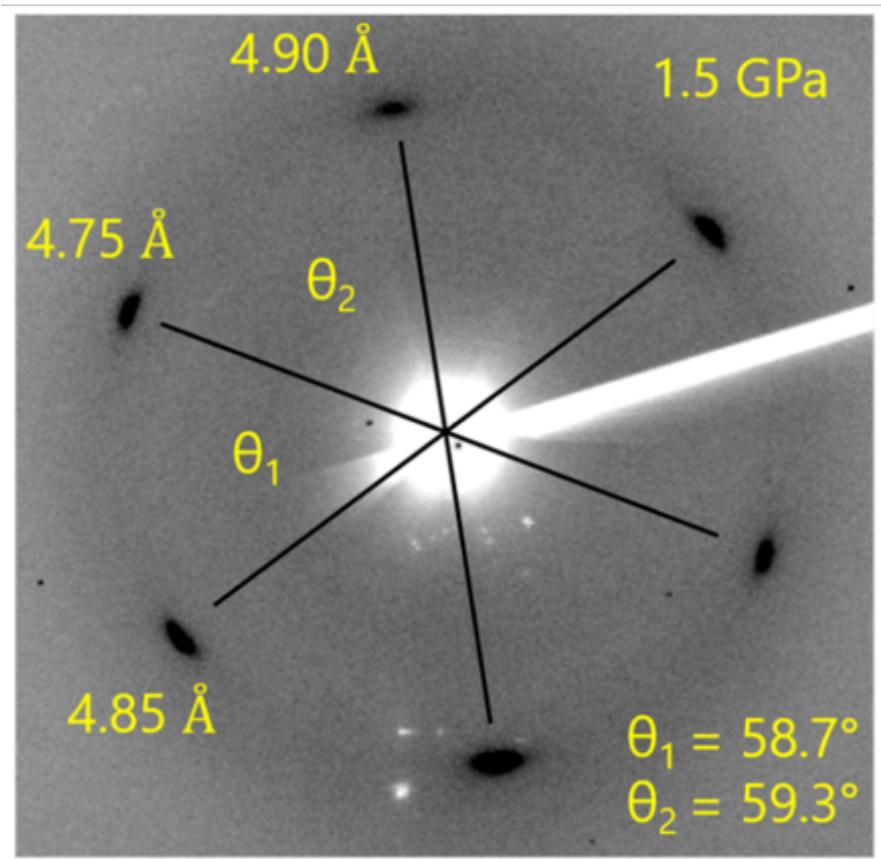

\section{simulation}

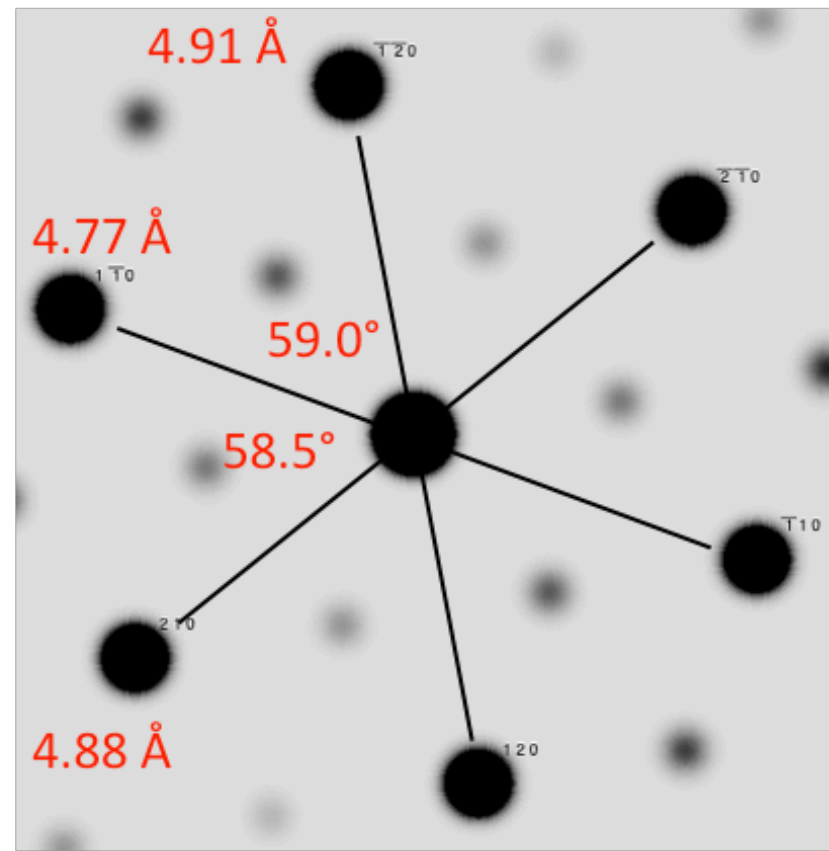

Figure S8. Experimental vs. simulated diffraction pattern (at $0 \mathrm{~K}$ ) of furan nanothreads. The C-2-a packing for the anti-thread was used in the simulation. 


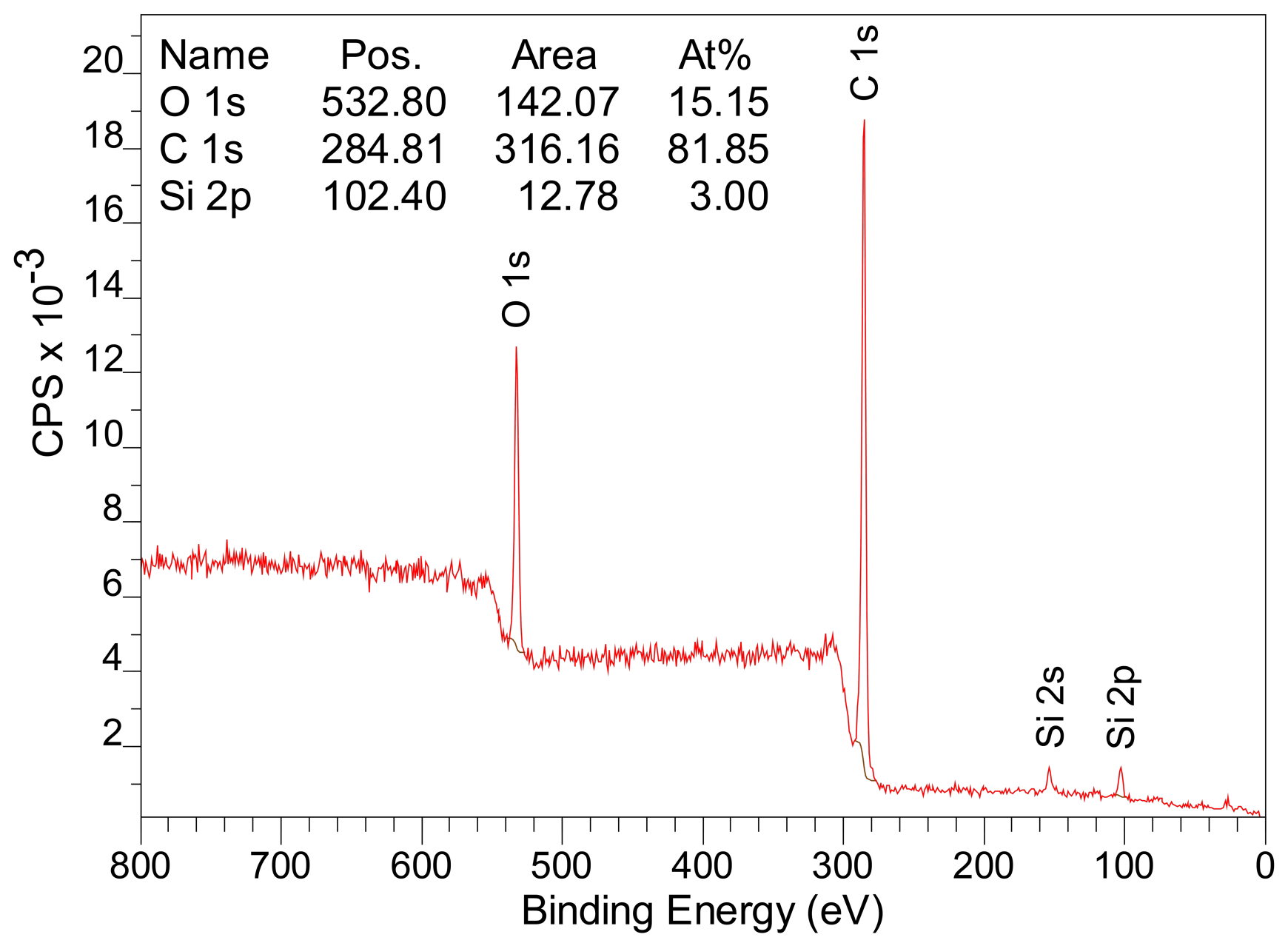

Figure S9. XPS survey scan of the recovered solid from the compression of furan.

The presence of silicon in the XPS survey scan was attributed to a monolayer of polydimethylsiloxane (PDMS). To account for this monolayer, we subtracted out the PDMS by using its base chemical formula $\left(\mathrm{SiOCH}_{3}\right)_{\mathrm{n}}$. The atomic percentages were then normalized, to give a $\mathrm{C}: \mathrm{O}$ ratio of $6.2: 1$. Assuming that this surface characterization represents the bulk of the solid, this is higher than the expected C:O ratio of 4:1. This shows that while furan nanothreads are likely formed, side reactions are likely occurring which may form products of varying carbon to oxygen ratios. These products may be volatile. 


\section{IR spectra simulation and comparison of peak positions with experiment}

Table S2. Comparison of Peak Positions in Experimental and Simulations

\begin{tabular}{|c|c|c|c|c|}
\hline Experimental & \multicolumn{3}{|c|}{ Calculated Frequencies $\left(\mathrm{cm}^{-1}\right)$} & \multirow[t]{2}{*}{ Assignment } \\
\hline This work & Syn & Anti & Syn/Anti & \\
\hline 478 & 483 & & & C-O-C wagging \\
\hline 530 & & $537(w)$ & 518 and $539(w)$ & C-O-C wagging \\
\hline 562 & & 587 & & C-O-C wagging \\
\hline 700 (shoulder) & $700(w)$ & & 697 & Ring breathing \\
\hline 720 & $711(\mathrm{w})$ & 715 & & Ring breathing \\
\hline 756 & 757 & & $744(w)$ & Ring breathing \\
\hline 785 & & 789 & 793 & C-H bending (tentative) \\
\hline 867 & $865(w)$ & 878 and 860 & $875(w)$ & $\mathrm{C}-\mathrm{H}$ bending (tentative) \\
\hline 909 & $910(w)$ & 885 & 895 and 903 & C-H bending (tentative) \\
\hline 931 & 922 & & $930(w)$ & $\mathrm{C}-\mathrm{H}$ bending (tentative) \\
\hline$\sim 965$ (shoulder) & 967 & 956 & 965 & $\mathrm{C}-\mathrm{H}$ bending (tentative) \\
\hline 1018 & 1011 & 1031 & 1019 & C-O stretching (tentative) \\
\hline 1213 & 1205 & 1217 & 1222 & C-H bending \\
\hline 1259 & $\begin{array}{l}1260 \\
(w)\end{array}$ & $1271(\mathrm{w})$ & 1269 & $\begin{array}{l}\mathrm{C}-\mathrm{O} \text { stretching and/or C-H } \\
\text { bending (tentative) }\end{array}$ \\
\hline 1305 & & $1300(w)$ & 1310 & $\begin{array}{l}\mathrm{C}-\mathrm{O} \text { stretching and/or C-H } \\
\text { bending (tentative) }\end{array}$ \\
\hline 2965 & 2955 & $\begin{array}{c}2965 \text { and } \\
2985\end{array}$ & 2970 & C-H stretching \\
\hline
\end{tabular}

Note: (w) denotes a weak intensity; otherwise, the signal is strong/medium. 


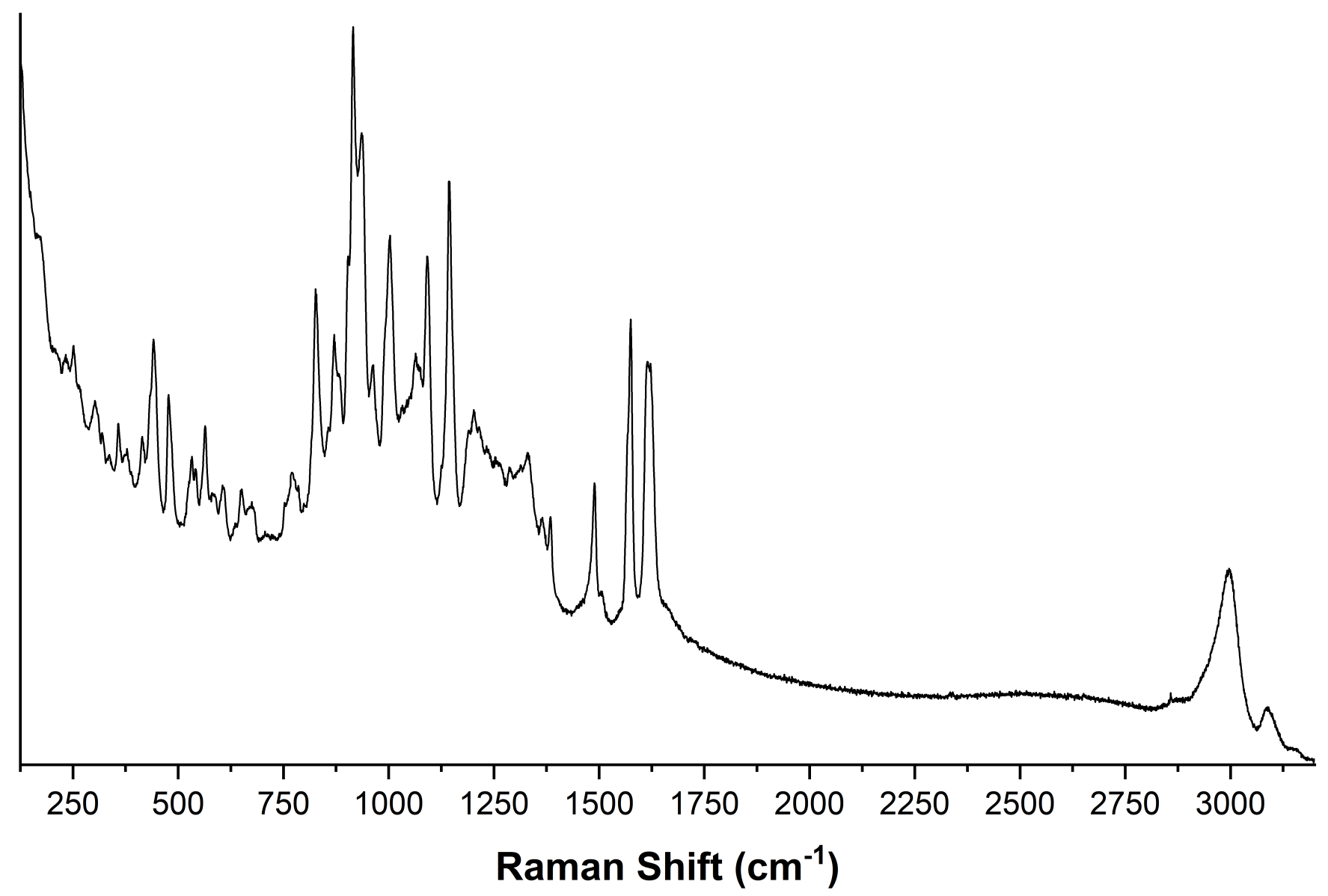

Figure S10. Raman spectrum of furan nanothreads produced in a PE Press; $785 \mathrm{~nm}$ excitation wavelength. 


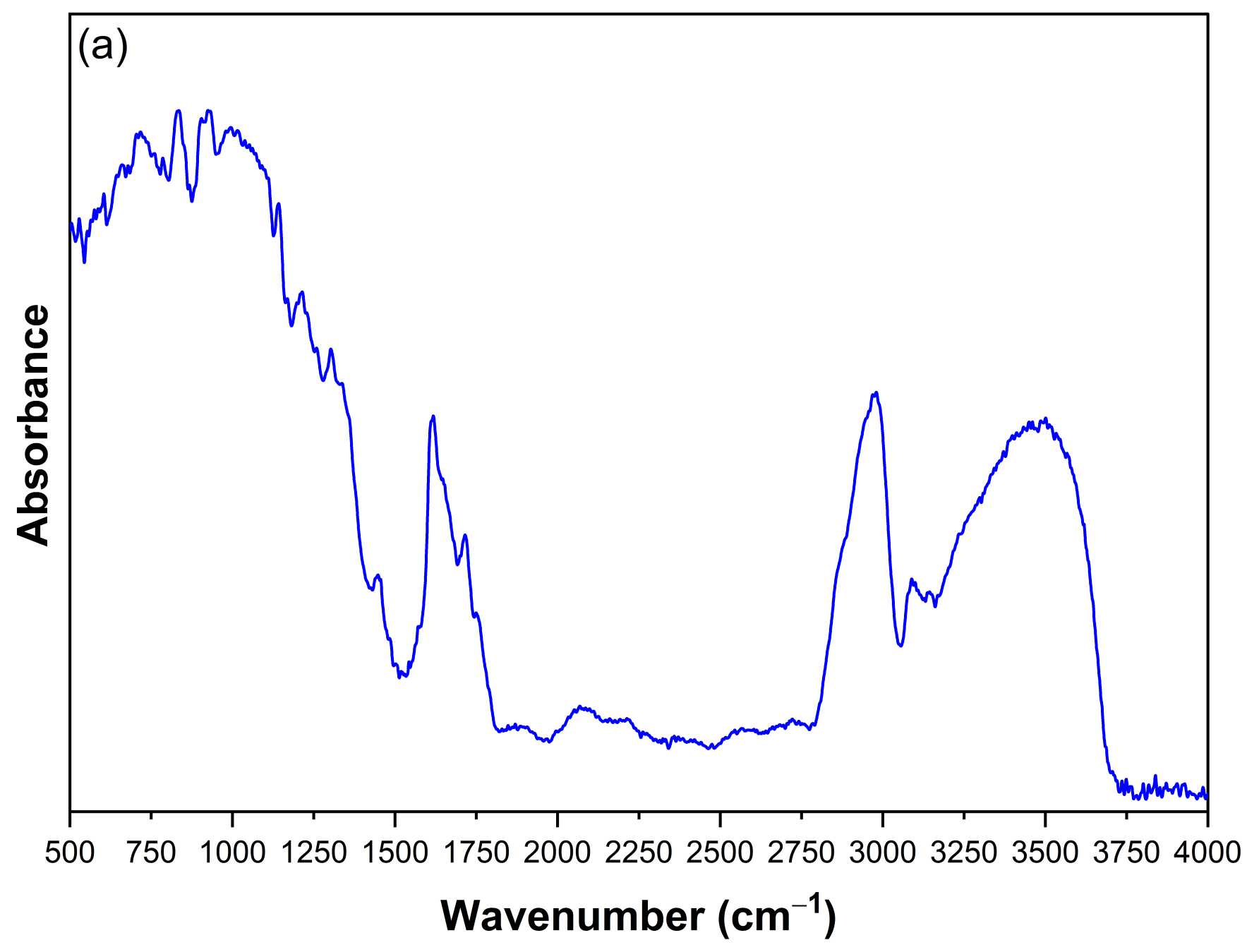

Figure S11. Reflectance Infrared Spectrum of recovered solid from the slow compression of furan in a PE Press. Note: reflectance IR was performed due to the sample thickness. 
(a)

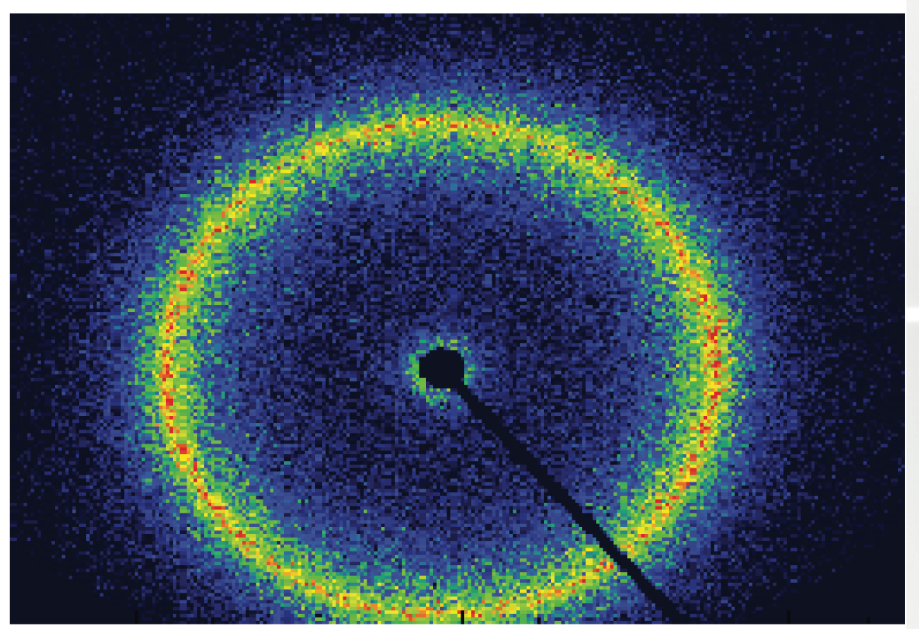

\section{d-spacing $=5.05 \AA$}

(b)

\section{d-spacing $=4.96 \AA$}

Figure S12. (a) Wide-angle X-Ray scattering and (b) In-House XRD two-dimensional X-Ray diffraction of recovered solid from slow compression of slow-compression-pressure-induced reaction of furan in a PE Press. 


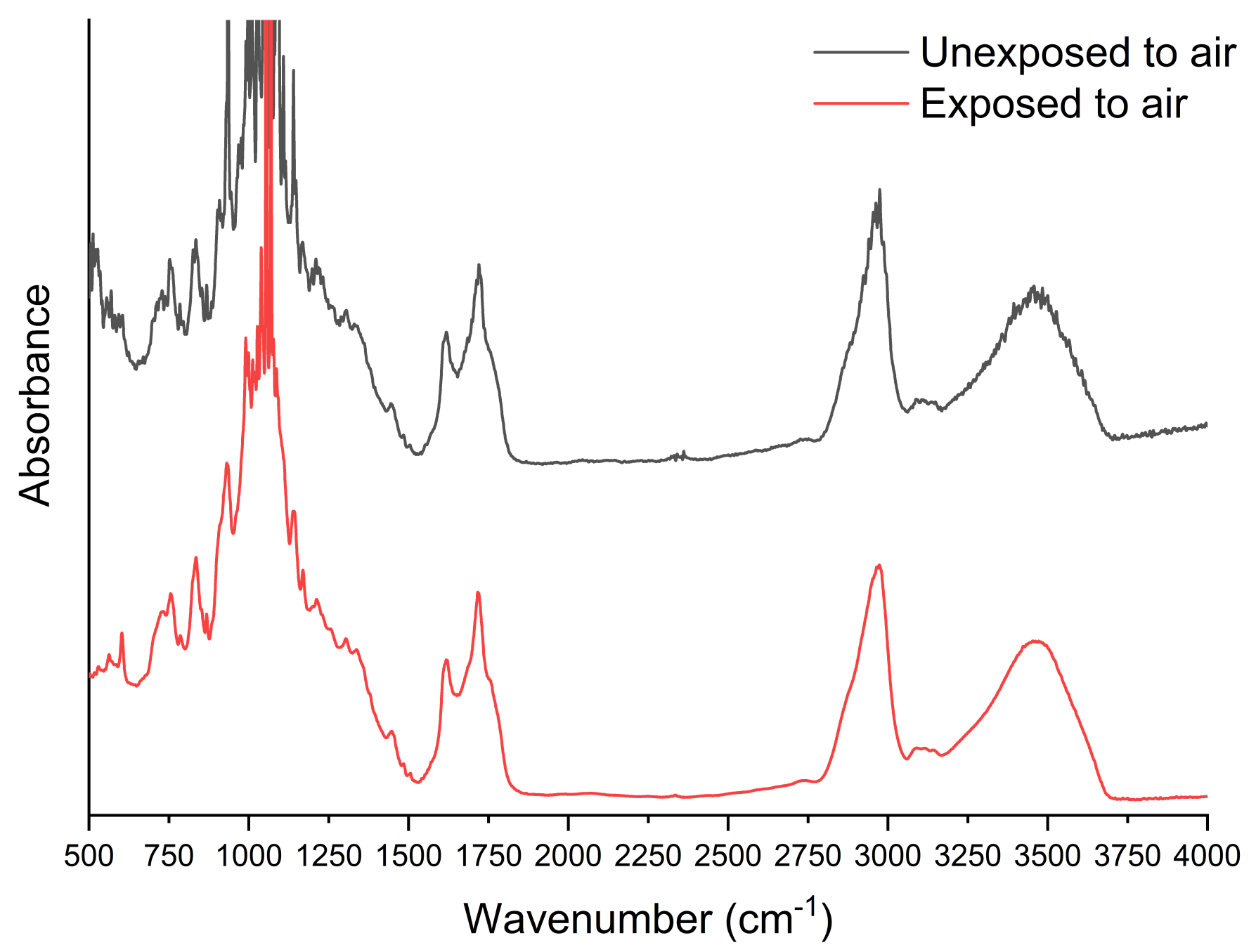

Figure S13. Infrared spectra of furan-derived nanothreads produced in a Diamond Anvil Cell unexposed to air and exposed to air. Due to sample thickness, signals at $\sim 1100 \mathrm{~cm}^{-1}$ are saturated. 


\section{Broadband FT-ICR MS of furan nanothreads from a PE Press}

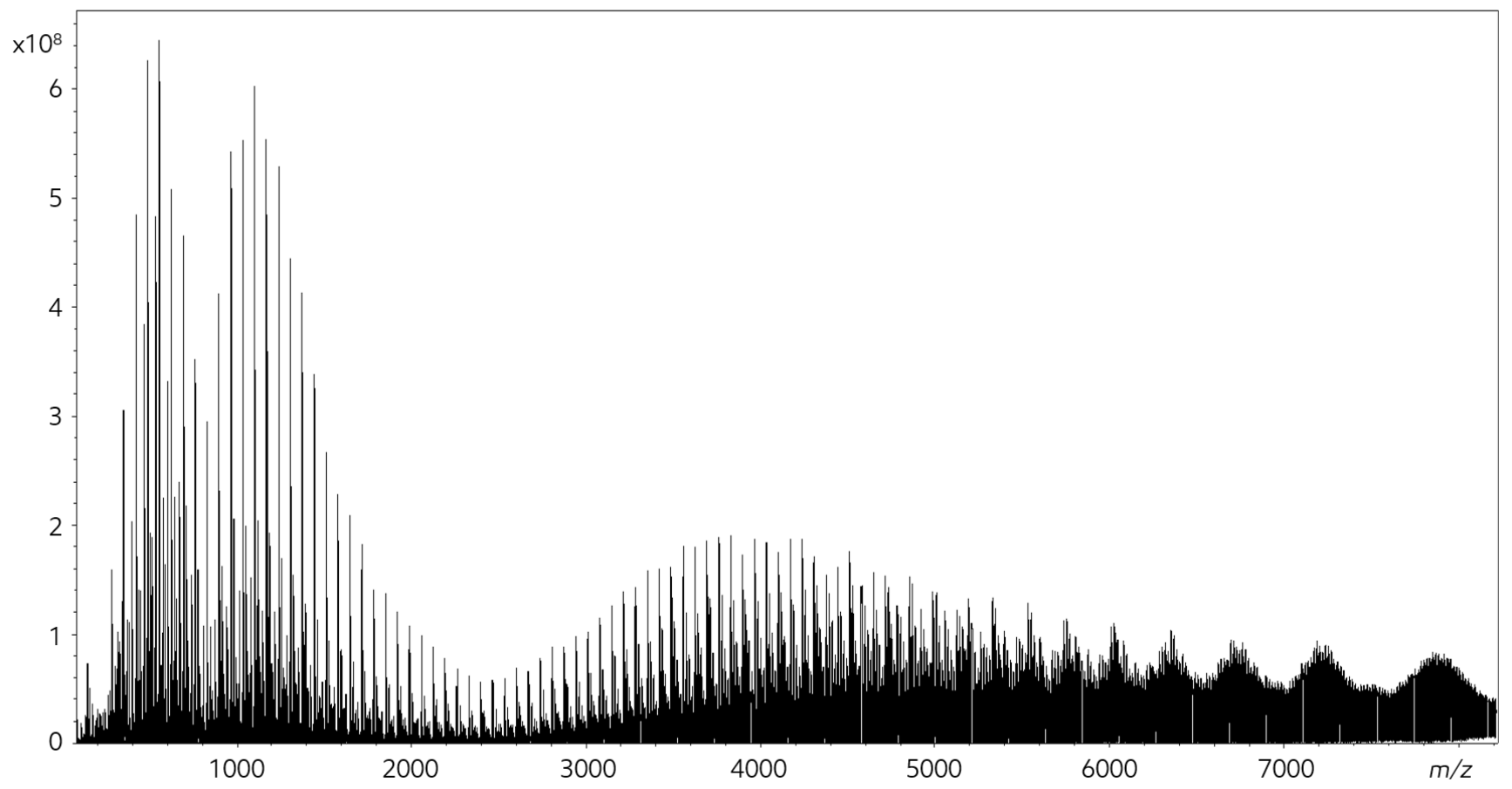

Figure S14. A broadband mass spectrum of the furan nanothreads was acquired using MALDI MS on an FT-ICR MS. A total of 3 mass spectra were acquired and overlaid to generate this single spectrum: 1) low-mass MS, 2) mid-mass MS, and 3) high-mass MS.

The high resolving power inherent to the FT-ICR MS is crucial for this type of analysis, and the higher mass peaks would likely go unresolved if another platform was used. The complexity of the nanothreads likely caused the irregular signal at the extremely high mass range at $>7 \mathrm{kDa}$, which appeared as small 'humps' of signal. In order to detect the higher mass components, another MS should be incorporated into the workflow. A Time-of-Flight (TOF) MS may provide the extended mass range necessary to generate a better understanding of the MW of the threads. With the FT-ICR alone, we cannot say with certainty if we are measuring fragments from larger threads, or the individual threads themselves.

\section{References}

(1) Guo, L.; Wang, G.; Yan, Z.; Zhang, X. Mechanism for Covalent Dimerization of Pyridine: [4+2] Dimerization, an MP2 Investigation. Chem. Phys. Lett. 2016, 644, 132-137.

(2) Grunbaum, B.; Shephard, G. C. Ellipse Patterns. Tilings and Patterns, $1^{\text {st }}$ ed; W. H. Freeman and Company: New York, 1987; pp 375-389. 